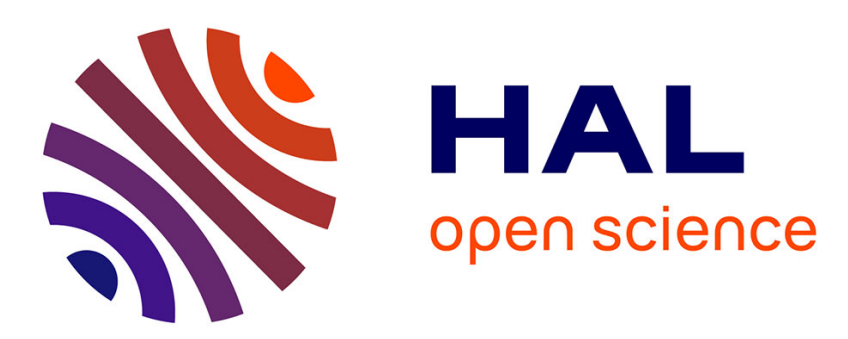

\title{
A moment approach for the almost sure central limit theorem for martingales
}

Bernard Bercu, Jean-Claude Fort

\section{To cite this version:}

Bernard Bercu, Jean-Claude Fort. A moment approach for the almost sure central limit theorem for martingales. Studia Scientiarum Mathematicarum Hungarica, 2008, 45, pp.139-159. hal-00012679

\section{HAL Id: hal-00012679 \\ https://hal.science/hal-00012679}

Submitted on 26 Oct 2005

HAL is a multi-disciplinary open access archive for the deposit and dissemination of scientific research documents, whether they are published or not. The documents may come from teaching and research institutions in France or abroad, or from public or private research centers.
L'archive ouverte pluridisciplinaire HAL, est destinée au dépôt et à la diffusion de documents scientifiques de niveau recherche, publiés ou non, émanant des établissements d'enseignement et de recherche français ou étrangers, des laboratoires publics ou privés. 


\title{
On Carleman approach for the almost sure central limit theorem for martingales
}

\author{
B. Bercu and J. C. Fort \\ Laboratoire de Statistique et Probabilités, UMR C5583, Université Paul Sabatier, \\ 118 Route de Narbonne, 31062 Toulouse Cedex, France. ${ }^{1}$
}

\begin{abstract}
We prove the almost sure central limit theorem for martingales via an original approach which uses the Carleman moment theorem together with the convergence of moments of martingales. Several statistical applications to autoregressive and branching processes are also provided.
\end{abstract}

Key words: Martingale transforms, Moments.

AMS Classification: 60G42, 60F05.

\section{Introduction.}

Let $\left(X_{n}\right)$ be a sequence of independent and identically distributed random variables with $\mathbb{E}\left[X_{n}\right]=0, \mathbb{E}\left[X_{n}^{2}\right]=\sigma^{2}$. The almost sure central limit theorem (ASCLT) associated with $\left(X_{n}\right)$ states that the empirical measure

$$
G_{n}=\frac{1}{\log n} \sum_{k=1}^{n} \frac{1}{k} \delta_{S_{k} / \sqrt{k}} \quad \text { with } \quad S_{n}=\sum_{k=1}^{n} X_{k}
$$

converges a.s. to the standard $\mathcal{N}\left(0, \sigma^{2}\right)$ distribution. It was simultaneously established by Brosambler [4] and Schatte [17], [18] and in the present form by Lacey and Phillip [12]. While a wide literature concerning the ASCLT for independent random variables is now available, very few references may be found on the ASCLT for martingales apart from the important contribution of Chaabane et al. [5], [6], [7] and Lifshits [14], [15]. Let $\left(\varepsilon_{n}\right)$ be a martingale difference sequence adapted to an appropriate filtration $\mathbb{F}=\left(\mathcal{F}_{n}\right)$ with $\mathbb{E}\left[\varepsilon_{n+1}^{2} \mid \mathcal{F}_{n}\right]=\sigma^{2}$

$\overline{1}$ E-mail: bercu@math.ups-tlse.fr and fort@math.ups-tlse.fr 
a.s. and denote by $\left(\phi_{n}\right)$ a sequence of random variables adapted to $\mathbb{F}$. We shall investigate the ASCLT for the real martingale transform $\left(M_{n}\right)$ given by

$$
M_{n}=\sum_{k=1}^{n} \phi_{k-1} \varepsilon_{k}
$$

The explosion coefficient associated with $\left(\phi_{n}\right)$

$$
f_{n}=\frac{\phi_{n}^{2}}{s_{n}} \quad \text { where } \quad s_{n}=\sum_{k=0}^{n} \phi_{k}^{2}
$$

will play a crucial role in all the sequel. Hereafter, we assume that $\left(s_{n}\right)$ increases a.s. to infinity. The more accurate ASCLT for martingales, due to Chaabane [5], is as follows.

Theorem 1. Let $\Delta M_{n}=M_{n}-M_{n-1}$ and denote by $\left(V_{n}\right)$ a positive predictable sequence such that

$$
\lim _{n \rightarrow \infty} V_{n}^{-2} s_{n-1}=1 \quad \text { a.s }
$$

For all $\varepsilon>0 \quad \sum_{n=1}^{\infty} V_{n}^{-2} \mathbb{E}\left[\Delta M_{n}^{2} \mathbb{I}_{\left(\left|\Delta M_{n}\right|>\varepsilon V_{n}\right)} \mid \mathcal{F}_{n-1}\right]<\infty$

$$
\text { For some } a>0 \quad \sum_{n=1}^{\infty} V_{n}^{-2 a} \mathbb{E}\left[\left|\Delta M_{n}\right|^{2 a} \mathbb{I}_{\left(\left|\Delta M_{n}\right| \leq V_{n}\right)} \mid \mathcal{F}_{n-1}\right]<\infty
$$

Then, $\left(M_{n}\right)$ satisfies the ASCLT

$$
\frac{1}{\log V_{n}^{2}} \sum_{k=1}^{n}\left(\frac{V_{k+1}^{2}-V_{k}^{2}}{V_{k+1}^{2}}\right) \delta_{M_{k} / V_{k}} \Longrightarrow G \quad \text { a.s. }
$$

where $G$ stands for the standard $\mathcal{N}\left(0, \sigma^{2}\right)$ distribution.

One can easily check that, under the assumptions of Theorem $1, V_{n+1}^{2}$ is a.s. equivalent to $V_{n}^{2}$ so that the explosion coefficient $f_{n}$ tends to zero a.s. In addition, the simple choice $V_{n}^{2}=s_{n-1}$ leads to

$$
\frac{1}{\log s_{n}} \sum_{k=1}^{n} f_{k} \delta_{M_{k} / \sqrt{s_{k-1}}} \Longrightarrow G \quad \text { a.s. }
$$

In other words, for any bounded continuous real function $h$

$$
\lim _{n \rightarrow \infty} \frac{1}{\log s_{n}} \sum_{k=1}^{n} f_{k} h\left(\frac{M_{k}}{\sqrt{s_{k-1}}}\right)=\int_{\mathbb{R}} h(x) d G(x) \quad \text { a.s. }
$$

In all what follows, we shall say that $\left(M_{n}\right)$ satisfies a polynomial almost sure central limit theorem (PASCLT) if convergence (1.5) holds for any polynomial 
function $h$ over $\mathbb{R}$. Of course, a PASCLT immediately implies a standard ASCLT. One might wonder if the theoretical study of ASCLT for martingales is completely acheived by Theorem 1 . To be more precise, is it possible to characterize the largest class of real martingale transforms satisfying (1.4)? As notice by Lifshits [14], the assumptions of Theorem 1 are too restrictive. For example, (1.2) is not satisfied for martingales with rare jumps of magnitude greater than $V_{n}$ as (1.2) immediately implies that, for all $\varepsilon>0$,

$$
\sum_{n=1}^{\infty} \mathbb{I}_{\left(\left|\Delta M_{n}\right|>\varepsilon V_{n}\right)}<\infty \quad \text { a.s }
$$

Moreover, one can realize that (1.3) does not hold for martingales with explosion coefficient $f_{n}$ decreasing slowly to zero. For example, assume that $\left(\varepsilon_{n}\right)$ are i.i.d. with symetric Bernoulli distribution and let $\left(\phi_{n}\right)$ be positive deterministic such that $\phi_{0}=1$ and for all $n \geq 1$

$$
\phi_{n}^{2}=\frac{1}{\log (e+n)} \prod_{k=1}^{n} \frac{\log (e+k)}{\log (e+k)-1} .
$$

Then, $\left(s_{n}\right)$ increases to infinity, $f_{n}$ tends to zero almost surely as

$$
s_{n}=\prod_{k=1}^{n} \frac{\log (e+k)}{\log (e+k)-1} \quad \text { and } \quad f_{n}=\frac{1}{\log (e+n)} .
$$

However, (1.3) always fails as it reduces to

$$
\sum_{n=0}^{\infty} f_{n}^{a}=\infty \quad \text { a.s }
$$

Nevertheless, we will show in the sequel that $\left(M_{n}\right)$ satisfies an ASCLT.

The paper is organized as follows. In section 2, we establish a new ASCLT based on the Carleman moment theorem together with the convergence of moments of martingales. Section 3 is devoted to similar results when the explosion coefficient $f_{n}$ converges a.s. to a positive random variable. Statistical applications to autoregressive and branching processes are developed in section 4, while all technical proofs are postponed in the Appendices.

\section{On Carleman approach.}

The classical moment problem concerns the question whether or not a given sequence of moments $\left(m_{n}\right)$ uniquely determines the associated probability distribution. One can find many probability distribution which are not uniquely 
determined by their moments, for example the log-normal distribution. However, the celebrated Carleman theorem gives a positive answer to that question under a suitable condition on the moments $\left(m_{n}\right)$.

Theorem 2. A probability distribution is uniquely determined by its moments $\left(m_{n}\right)$ if

$$
\sum_{n=1}^{\infty} m_{2 n}^{-1 / 2 n}=\infty
$$

We will make use of this result in the martingale framework via the following theorem where the first convergence (2.3) was recently proven in [2].

Theorem 3. Assume that $\left(\varepsilon_{n}\right)$ is a martingale difference sequence such that $\mathbb{E}\left[\varepsilon_{n+1}^{2} \mid \mathcal{F}_{n}\right]=\sigma^{2}$ a.s. and satisfying, for some integer $p \geq 1$ and some real $a>2 p$, the moment condition

$$
\sup _{n \geq 0} \mathbb{E}\left[\left|\varepsilon_{n+1}\right|^{a} \mid \mathcal{F}_{n}\right]<\infty \quad \text { a.s. }
$$

In addition, assume that the explosion coefficient $f_{n}$ tends to zero a.s. Then

$$
\lim _{n \rightarrow \infty} \frac{1}{\log s_{n}} \sum_{k=1}^{n} f_{k}\left(\frac{M_{k}}{\sqrt{s_{k-1}}}\right)^{2 p}=\frac{\sigma^{2 p}(2 p) !}{2^{p} p !} \quad \text { a.s. }
$$

In addition, we also have

$$
\lim _{n \rightarrow \infty} \frac{1}{\log s_{n}} \sum_{k=1}^{n} f_{k}\left(\frac{M_{k}}{\sqrt{s_{k-1}}}\right)^{2 p-1}=0 \quad \text { a.s. }
$$

One can observe that the Gaussian limit distribution clearly satisfies Carleman's moment condition (2.1). Gathering the last two theorems, we deduce the following PASCLT for martingales.

Theorem 4. Assume that $\left(\varepsilon_{n}\right)$ is a martingale difference sequence such that $\mathbb{E}\left[\varepsilon_{n+1}^{2} \mid \mathcal{F}_{n}\right]=\sigma^{2}$ a.s. and satisfying, for all integer $p \geq 1$,

$$
\sup _{n \geq 0} \mathbb{E}\left[\left|\varepsilon_{n+1}\right|^{p} \mid \mathcal{F}_{n}\right]<\infty \quad \text { a.s. }
$$

In addition, assume that the explosion coefficient $f_{n}$ tends to zero a.s. Then, the martingale transform $\left(M_{n}\right)$ satisfies the PASCLT given by (1.5).

Proof. For all $n \geq 1$, define the empirical measure

$$
G_{n}=\frac{1}{\log s_{n}} \sum_{k=1}^{n} f_{k} \delta_{M_{k} / \sqrt{s_{k-1}}} .
$$


For all integer $p \geq 0$, we obviously have

$$
\sup _{p \geq 0} \int_{\mathbb{R}} x^{2 p} d G_{n}(x)<\infty \quad \text { a.s. }
$$

This is a well-known condition ensuring the tightness of the sequence $\left(G_{n}\right)$ (see e.g. Duflo [8] Proposition 2.1.6 p. 41). As the Gaussian limit distribution satisfies $(2.1),\left(G_{n}\right)$ weakly converges a.s. toward the standard $\mathcal{N}\left(0, \sigma^{2}\right)$ distribution which completes the proof of Theorem 4 .

Consider once again the enlightening example of the introduction where $\left(\varepsilon_{n}\right)$ are i.i.d. with symetric Bernoulli distribution and $\left(\phi_{n}\right)$ satisfies (1.6). The moment condition (2.5) clearly holds for all integer $p \geq 1$ since $\left(\varepsilon_{n}\right)$ is bounded by one. Moreover, $f_{n}$ decreases to zero a.s. with a logarithmic rate of convergence. Consequently, $\left(M_{n}\right)$ satisfies the PASCLT given by (1.5).

\section{Extension to explosive martingales.}

One might wonder whether or not an ASCLT holds when $f_{n}$ converges a.s. to a positive random variable $f$. Our goal is now to show that this is the case. First of all, we need an asymptotic result for the moments similar to that of Theorem 3. For any integer $p \geq 1$, set

$$
\sigma_{n}(p)=\mathbb{E}\left[\varepsilon_{n+1}^{p} \mid \mathcal{F}_{n}\right] .
$$

Theorem 5. Assume that $\left(\varepsilon_{n}\right)$ is a martingale difference sequence such that $\mathbb{E}\left[\varepsilon_{n+1}^{2} \mid \mathcal{F}_{n}\right]=\sigma^{2}$ a.s. and satisfying, for some integer $p \geq 1$, the moment condition (2.2). In addition, suppose that for any $2 \leq q \leq 2 p$,

$$
\lim _{n \rightarrow \infty} \sigma_{n}(q)=\sigma(q) \quad \text { a.s. }
$$

where $\sigma(q)=0$ if $q$ is odd. Moreover, assume that the explosion coefficient $f_{n}$ converges a.s. to a random variable $f$ with $0<f<1$. Then

$$
\begin{aligned}
& \lim _{n \rightarrow \infty} \frac{1}{n} \sum_{k=1}^{n}\left(\frac{M_{k}}{\sqrt{s_{k-1}}}\right)^{2 p}=l(p, f) \quad \text { a.s. } \\
& \lim _{n \rightarrow \infty} \frac{1}{n} \sum_{k=1}^{n}\left(\frac{M_{k}}{\sqrt{s_{k-1}}}\right)^{2 p-1}=0 \quad \text { a.s. }
\end{aligned}
$$

where $l(0, f)=1$ and, for $p \geq 1, l(p, f)$ satisfies the recurrence equation

$$
l(p, f)=\frac{1}{1-(1-f)^{p}} \sum_{k=1}^{p} C_{2 p}^{2 k} f^{k}(1-f)^{p-k} \sigma(2 k) l(p-k, f) .
$$


We now propose a non Gaussian ASCLT for explosive martingales.

Theorem 6. Assume that $\left(\varepsilon_{n}\right)$ is a martingale difference sequence such that $\mathbb{E}\left[\varepsilon_{n+1}^{2} \mid \mathcal{F}_{n}\right]=\sigma^{2}$ a.s. and satisfying, for all integer $p \geq 1$, the moment condition (2.5). and (3.1). In addition, assume that the explosion coefficient $f_{n}$ converges a.s. towards a random variable $f$ with $0<f<1$, and that the sequence $(l(p, f))$ satisfies Carleman's moment condition. Then, there exists a unique probability distribution $\mathcal{H}_{f}$ such that

$$
\frac{1}{n} \sum_{k=1}^{n} \delta_{M_{k} / \sqrt{s_{k-1}}} \Longrightarrow \mathcal{H}_{f} \quad \text { a.s. }
$$

Moreover, if the limiting moments sequence $(\sigma(p))$ define a probability distribution with Laplace transform $L_{\sigma}$ finite on a neighborhood of the origin, then the Laplace transform $L_{\mathcal{H}_{f}}$ of $\mathcal{H}_{f}$ exists a.s. on a neighborhood of the origin and is given by

$$
L_{\mathcal{H}_{f}}(t)=\prod_{k=0}^{\infty} L_{\sigma}\left(f^{1 / 2}(1-f)^{k / 2} t\right) \quad \text { a.s. }
$$

Remark 7. On the one hand, an easy sufficient condition which ensures that the sequence $(l(p, f))$ satisfies Carleman's moment condition is that there exists some constant $C>0$ such that

$$
\sigma(2 p)=O\left(C^{p} p^{2 p}\right)
$$

On the other hand, one can see that (3.5) holds for any polynomial function. In addition, if all the moments $\sigma(2 p)$ coincide with those of an $\mathcal{N}\left(0, \sigma^{2}\right)$ random variable, then $\mathcal{H}_{f}$ is simply the $\mathcal{N}\left(0, \sigma^{2}\right)$ distribution.

Lastly, set $r=(1-f)^{-1 / 2}$ and assume that $r$ is an integer. From equation (3.6), it follows that $\mathcal{H}_{f}$ has the same distibution as

$$
\left(1-\frac{1}{r^{2}}\right)^{1 / 2} \sum_{k=0}^{\infty} \frac{\xi_{k}}{r^{k}}
$$

where the $\xi_{k}$ are independant random variables with moments $\sigma(p)$. Let $\left(B_{n}\right)$ be a sequence of independant random variables uniformly distributed over $\{0,1, \cdots, r-1\}$. If we choose $\xi_{k}=2 B_{k}-(r-1)$, then $\mathcal{H}_{f}$ coincides with the uniform distibution on the interval $\left[-\left(r^{2}-1\right)^{1 / 2},\left(r^{2}-1\right)^{1 / 2}\right]$ (see e.g. [8] page 44). As a matter of fact, $\mathcal{H}_{f}$ shares the same distribution as

$$
\left(1-\frac{1}{r^{2}}\right)^{1 / 2} \sum_{k=0}^{\infty} \frac{\xi_{k}}{r^{k}}=\left(1-\frac{1}{r^{2}}\right)^{1 / 2}\left(2 \sum_{k=0}^{\infty} \frac{B_{k}}{r^{k}}-r\right)
$$

Proof. We obtain convergence (3.5) proceeding exactly as in the proof of Theorem 4. Hence, it only remains to prove relation (3.6). We introduce the 
following Laplace transforms or moment generating functions as extended real numbers

$$
L_{\mathcal{H}_{f}}(t)=\sum_{p=0}^{\infty} \frac{l(p, f)}{(2 p) !} t^{2 p} \quad \text { and } \quad L_{\sigma}(t)=\sum_{p=0}^{\infty} \frac{\sigma(2 p)}{(2 p) !} t^{2 p} .
$$

One can observe that if $L_{\sigma}$ is finite on a neighborhood of the origin, then $\sigma(2 p)=O\left(C^{p} p^{2 p}\right)$ for some constant $C>0$. Then, we easily deduce from equation (3.4) that $l(p, f)=O\left(D^{p} p^{2 p}\right)$ for some other constant $D>0$ which yields the existence of $L_{\mathcal{H}_{f}}$ on a neighborhood of zero. Using again formula (3.4), we obtain that

$$
\begin{aligned}
L_{\mathcal{H}_{f}}(t) & =\sum_{p=0}^{\infty} \frac{t^{2 p}}{(2 p) !} \sum_{k=0}^{p} C_{2 p}^{2 k} f^{k}(1-f)^{p-k} \sigma(2 k) l(p-k, f), \\
& =\sum_{k=0}^{\infty} \frac{\sigma(2 k)}{(2 k) !} f^{k} t^{2 k} \sum_{p=k}^{\infty} \frac{l(p-k, f)}{(2(p-k)) !}(1-f)^{p-k} t^{2(p-k)} \\
& =L_{\sigma}\left(f^{1 / 2} t\right) \sum_{p=0}^{\infty} \frac{l(p, f)}{(2 p) !}\left((1-f)^{1 / 2} t\right)^{p} \\
& =L_{\sigma}\left(f^{1 / 2} t\right) L_{\mathcal{H}_{f}}\left((1-f)^{1 / 2} t\right),
\end{aligned}
$$

which immediately leads to (3.6), completing the proof of Theorem 6 .

\section{Applications.}

\subsection{Linear regressions.}

Consider the stochastic linear regression model given by

$$
X_{n}=\theta \phi_{n-1}+\varepsilon_{n}
$$

where $X_{n}$ and $\phi_{n}$ are the observation and the regression variable, respectively. We assume that the driven noise $\left(\varepsilon_{n}\right)$ is a martingale difference sequence such that $\mathbb{E}\left[\varepsilon_{n+1}^{2} \mid \mathcal{F}_{n}\right]=\sigma^{2}$ a.s. In order to estimate the unknown real parameter $\theta$, we shall make use of the least squares estimator $\widehat{\theta}_{n}$ which satisfies

$$
\widehat{\theta}_{n}-\theta=\frac{M_{n}}{s_{n-1}} \quad \text { where } \quad M_{n}=\sum_{k=1}^{n} \phi_{k-1} \varepsilon_{k} .
$$

A straightforward application of Theorem 4 is as follows.

Corollary 8. Assume that $\left(\varepsilon_{n}\right)$ is a martingale difference sequence satisfying, for all integer $p \geq 1$, the moment condition (2.5). In addition, suppose that 
$s_{n}$ increases a.s. to infinity and that $f_{n}$ converge a.s. towards zero. Then, $\left(\widehat{\theta}_{n}\right)$ satisfies the PASCLT

$$
\frac{1}{\log s_{n}} \sum_{k=1}^{n} f_{k} \delta_{\sqrt{s_{k}}\left(\widehat{\theta}_{k}-\theta\right)} \Longrightarrow \mathcal{N}\left(0, \sigma^{2}\right) \quad \text { a.s. }
$$

More particularly, assume that for some positive constant $\tau$

$$
\lim _{n \rightarrow \infty} \frac{s_{n}}{n}=\tau \quad \text { a.s. }
$$

Then, we have the PASCLT

$$
\frac{1}{\log n} \sum_{k=1}^{n} \frac{1}{k} \delta_{\sqrt{k}\left(\widehat{\theta}_{k}-\theta\right)} \Longrightarrow \mathcal{N}\left(0, \frac{\sigma^{2}}{\tau}\right) \quad \text { a.s. }
$$

Remark 9. We immediately infer from (4.3) that for all integer $p \geq 1$

$$
\lim _{n \rightarrow \infty} \frac{1}{\log n} \sum_{k=1}^{n} k^{p-1}\left(\widehat{\theta}_{k}-\theta\right)^{2 p}=\frac{\sigma^{2 p}(2 p) !}{\tau^{p} 2^{p} p !} \quad \text { a.s. }
$$

while

$$
\lim _{n \rightarrow \infty} \frac{1}{\log n} \sum_{k=1}^{n} k^{p-3 / 2}\left(\widehat{\theta}_{k}-\theta\right)^{2 p-1}=0 \quad \text { a.s. }
$$

The simple choice $\phi_{n}=X_{n}$ in (4.1) leads to the linear autoregressive model

$$
X_{n}=\theta X_{n-1}+\varepsilon_{n}
$$

In the stable case $|\theta|<1$, it is well-known that $f_{n}$ tends a.s. to zero and $s_{n} / n$ converges a.s. to $\sigma^{2} /\left(1-\theta^{2}\right)$ (see e.g. [8], [13], [19]). Hence, it follows from (4.3) that $\left(\widehat{\theta}_{n}\right)$ satisfies the PASCLT

$$
\frac{1}{\log n} \sum_{k=1}^{n} \frac{1}{k} \delta_{\sqrt{k}\left(\widehat{\theta}_{k}-\theta\right)} \Longrightarrow \mathcal{N}\left(0,1-\theta^{2}\right) \quad \text { a.s. }
$$

In the unstable case $|\theta|=1$, once again $f_{n} \rightarrow 0$ but $s_{n} / n^{2}$ diverges. However, by formula (3.5) of Wei [19], $\log s_{n}$ is a.s. equivalent to $2 \log n$. Consequently, only (4.2) holds replacing $\log s_{n}$ by $2 \log n$.

Similarly to Corollary 8, a direct application of Theorem 6 for explosive martingales is as follows.

Corollary 10. Assume that $\left(\varepsilon_{n}\right)$ is a martingale difference sequence satisfying, for all integer $p \geq 1$, the moment condition (2.5) and (3.1). In addition, assume that the explosion coefficient $f_{n}$ converges a.s. towards a random variable $f$ with $0<f<1$, and that the sequence $(l(p, f))$ satisfies Carleman's 
moment condition. Then, $\left(\widehat{\theta}_{n}\right)$ satisfies the PASCLT

$$
\frac{1}{n} \sum_{k=1}^{n} \delta_{\sqrt{s_{k-1}}\left(\widehat{\theta}_{k}-\theta\right)} \Longrightarrow \mathcal{H}_{f} \quad \text { a.s. }
$$

In addition, assume that for some positive random variable $\tau$

$$
\lim _{n \rightarrow \infty}(1-f)^{n} s_{n}=\tau \quad \text { a.s. }
$$

Then, there exists a unique probability distribution $\mathcal{H}_{f}(\tau)$ such that

$$
\frac{1}{n} \sum_{k=1}^{n} \delta_{\left(\widehat{\theta}_{k}-\theta\right) /(1-f)^{k / 2}} \Longrightarrow \mathcal{H}_{f}(\tau) \quad \text { a.s. }
$$

Lastly, if all the moments $\sigma(2 p)$ coincide with those of an $\mathcal{N}\left(0, \sigma^{2}\right)$ random variable, then we have the PASCLT

$$
\frac{1}{n} \sum_{k=1}^{n} \delta_{\left(\widehat{\theta}_{k}-\theta\right) /(1-f)^{k / 2}} \Longrightarrow \mathcal{N}\left(0, \frac{\sigma^{2}}{\tau(1-f)}\right) \quad \text { a.s. }
$$

Remark 11. As (4.6) holds for any polynomial function, We find that for all integer $p \geq 1$

$$
\lim _{n \rightarrow \infty} \frac{1}{n} \sum_{k=1}^{n} \frac{\left(\hat{\theta}_{k}-\theta\right)^{2 p}}{(1-f)^{k p}}=\frac{l(p, f)}{\tau^{p}(1-f)^{p}} \quad \text { a.s. }
$$

while

$$
\lim _{n \rightarrow \infty} \frac{1}{n} \sum_{k=1}^{n} \frac{\left(\widehat{\theta}_{k}-\theta\right)^{2 p-1}}{(1-f)^{k(p-1 / 2)}}=0 \quad \text { a.s. }
$$

Consider once again the linear autoregressive model given by (4.4). In the explosive case $|\theta|>1, \theta^{-n} X_{n}$ converges a.s. and in mean square to the positive random variable

$$
Y=X_{0}+\sum_{k=1}^{\infty} \theta^{-k} \varepsilon_{k} .
$$

Hence, it follows from Toeplitz's lemma that $f_{n} \rightarrow\left(\theta^{2}-1\right) / \theta^{2}$ a.s. and $s_{n} / \theta^{2 n}$ converges a.s. to $\theta^{2} Y^{2} /\left(\theta^{2}-1\right)$ (see e.g. [8], [13]). Consequently, we deduce from (4.6) that

$$
\frac{1}{n} \sum_{k=1}^{n} \delta_{|\theta|^{k}\left(\widehat{\theta}_{k}-\theta\right)} \Longrightarrow \mathcal{H}_{f}(\tau) \quad \text { a.s. }
$$

with $\tau=\theta^{2} Y^{2} /\left(\theta^{2}-1\right)$. More particularly, if all the moments $\sigma(2 p)$ coincide with those of an $\mathcal{N}\left(0, \sigma^{2}\right)$ random variable, we have the PASCLT

$$
\frac{1}{n} \sum_{k=1}^{n} \delta_{|\theta|^{k}\left(\widehat{\theta}_{k}-\theta\right)} \Longrightarrow \mathcal{N}\left(0, \frac{\sigma^{2}\left(\theta^{2}-1\right)}{Y^{2}}\right) \quad \text { a.s. }
$$




\subsection{Branching processes.}

Consider the Galton-Watson process given by

$$
X_{n}=\sum_{k=1}^{X_{n-1}} Y_{n, k}
$$

with $X_{0}=1$. The random variable $X_{n}$ denotes the size of the $n$-th generation while $Y_{n, k}$ is the number of offsprings of the $k$-th individual in the $(n-1)$-th generation. We assume that $\left(Y_{n, k}\right)$ is a sequence of independant and identically distributed random variables taking their values in $\mathbb{N}^{*}$, which means that $Y_{n, k} \geq 1$. The distribution of $\left(Y_{n, i}\right)$, with finite mean $m$ and positive variance $\sigma^{2}$, is commonly called the offspring distribution. We also suppose that $\left(Y_{n, i}\right)$ has finite moments of any order. Relation (4.7) can be rewritten as

$$
X_{n}=m X_{n-1}+\xi_{n}
$$

where $\xi_{n}=X_{n}-\mathbb{E}\left[X_{n} \mid \mathcal{F}_{n-1}\right]$. If

$$
\varepsilon_{n}=\frac{\xi_{n}}{\sqrt{X_{n-1}}}
$$

we clearly have $\mathbb{E}\left[\varepsilon_{n+1} \mid \mathcal{F}_{n}\right]=0$ and $\mathbb{E}\left[\varepsilon_{n+1}^{2} \mid \mathcal{F}_{n}\right]=\sigma^{2}$ a.s. The conditional least square estimator of $m$ is given by

$$
\widehat{m}_{n}=\frac{\sum_{k=1}^{n} X_{k}}{\sum_{k=1}^{n} X_{k-1}} .
$$

Consequently, we obtain from (4.8) that

$$
\widehat{m}_{n}-m=\frac{M_{n}}{s_{n-1}} \quad \text { where } \quad M_{n}=\sum_{k=1}^{n} \phi_{k-1} \varepsilon_{k}
$$

and $\phi_{n}=\sqrt{X_{n}}$. In the supercritical case $m>1$, it is well-known that $m^{-n} X_{n}$ converges a.s. and in mean square to the nonzero random variable

$$
L=X_{0}+\sum_{k=1}^{\infty} m^{-k} \xi_{k}
$$

Thus, we deduce from Toeplitz's lemma that $f_{n} \rightarrow(m-1) / m$ a.s. and $s_{n} / m^{n}$ converges a.s. to $m L /(m-1)$ (see e.g. [9]). Our purpose is now to propose a second application of Theorem 6 to $\left(\widehat{m}_{n}\right)$. Since $\left(Y_{n, k}\right)$ has finite moments of any order, the same remains true for the sequence $\left(\varepsilon_{n}\right)$. Hence, in order to make use of Theorem 6 , it is enough to verify the convergence of the conditional 
moments associated with $\left(\varepsilon_{n}\right)$. However, it follows from (4.7) that

$$
\varepsilon_{n}=\frac{\sum_{k=1}^{X_{n-1}}\left(Y_{n, k}-m\right)}{\sqrt{X_{n-1}}} .
$$

Consequently, applying the standard central limit theorem, the distribution of $\varepsilon_{n+1}$ conditionally to $\mathcal{F}_{n}$ converges to the gaussian distribution $\mathcal{N}\left(0, \sigma^{2}\right)$. For any $p \geq 1$, as the moments of order $2 p$ of $\varepsilon_{n+1}$ conditionally to $\mathcal{F}_{n}$ are bounded, a classsical argument of uniform integrability (see e.g. [3], theorem 25. 12) leads to the convergence of these moments to those of the $\mathcal{N}\left(0, \sigma^{2}\right)$ distribution. Therefore, a straightforward application of Theorem 6, similar to Corollary 10, is as follows.

Corollary 12. In the supercritical case $m>1,\left(\widehat{m}_{n}\right)$ satisfies the PASCLT

$$
\frac{1}{n} \sum_{k=1}^{n} \delta_{\sqrt{s_{k-1}}\left(\widehat{m}_{k}-m\right)} \Longrightarrow \mathcal{N}\left(0, \sigma^{2}\right) \quad \text { a.s. }
$$

Moreover, we also have

$$
\frac{1}{n} \sum_{k=1}^{n} \delta_{m^{k / 2}\left(\widehat{m}_{k}-m\right)} \Longrightarrow \mathcal{N}\left(0, \frac{(m-1) \sigma^{2}}{L}\right) \quad \text { a.s. }
$$

Remark 13. A standard ASCLT can be found in [16]. In addition, we infer from (4.10) that for all integer $p \geq 1$

$$
\lim _{n \rightarrow \infty} \frac{1}{n} \sum_{k=1}^{n} m^{k p}\left(\widehat{m}_{k}-m\right)^{2 p}=\frac{(m-1)^{p} \sigma^{2 p}(2 p) !}{L^{p} 2^{p} p !} \quad \text { a.s. }
$$

while

$$
\lim _{n \rightarrow \infty} \frac{1}{n} \sum_{k=1}^{n} m^{k(p-1 / 2)}\left(\widehat{m}_{k}-m\right)^{2 p-1}=0 \quad \text { a.s. }
$$

\section{Appendix A.}

Appendix A is devoted to the proof of Theorem 3. We shall only prove convergence for odd moments (2.4) as convergence for even moments (2.3) was already established in [2]. First of all, for any $p \geq 1$, set

$$
v_{n}(p)=\frac{\left(\sqrt{s_{n}}\right)^{2 p-1}-\left(\sqrt{s_{n-1}}\right)^{2 p-1}}{\left(\sqrt{s_{n}}\right)^{2 p-1}} .
$$

As $M_{n+1}=M_{n}+\phi_{n} \varepsilon_{n+1}$, we have for any $p \geq 1$

$$
M_{n+1}^{2 p-1}=\sum_{k=0}^{2 p-1} C_{2 p-1}^{k} \phi_{n}^{k} \varepsilon_{n+1}^{k} M_{n}^{2 p-1-k} .
$$


Consequently, putting

$$
V_{n}=\left(\frac{M_{n}}{\sqrt{s_{n-1}}}\right)^{2 p-1}
$$

we easily deduce from (A.1) that for any $n \geq 1$

$$
V_{n+1}+\mathcal{A}_{n}=V_{1}+\mathcal{B}_{n+1}+\mathcal{W}_{n+1}
$$

where

$$
\begin{array}{cc}
\mathcal{A}_{n}=\sum_{k=1}^{n} v_{k}(p) V_{k}, & \mathcal{W}_{n+1}=\sum_{k=1}^{n} s_{k}^{-p} \sqrt{s_{k}} \phi_{k}^{2 p-1} \varepsilon_{k+1}^{2 p-1}, \\
\mathcal{B}_{n+1}=\sum_{l=1}^{2 p-2} C_{2 p-1}^{l} B_{n+1}(l), & B_{n+1}(l)=\sum_{k=1}^{n} \varphi_{k}(l) \varepsilon_{k+1}^{l},
\end{array}
$$

and for any $1 \leq l \leq 2(p-1), \varphi_{k}(l)=s_{k}^{-p} \sqrt{s_{k}} M_{k}^{2 p-1-l} \phi_{k}^{l}$. Via a standard truncation argument, we may assume without loss of generality that each $\varphi_{k}(l)$ is a bounded random variable. Hereafter, by use of (A.2), we are in position to prove convergence (2.4) by induction on the power $p \geq 1$. For $p=1$, the term $\mathcal{B}_{n+1}$ in the right-hand side of (A.2) vanishes. In addition, it is well-known from [2], [8] or [13] that $M_{n}^{2}=O\left(s_{n-1} \log s_{n-1}\right)$ a.s. so that $V_{n+1}=o\left(\log s_{n}\right)$ a.s. Moreover, $\left(\mathcal{W}_{n}\right)$ is a locally square integrable martingale with increasing process

$$
<\mathcal{W}>_{n+1}=\sigma^{2} \sum_{k=1}^{n} f_{k}
$$

By the elementary inequality $x \leq-\log (1-x)$ for $0<x<1$, we have for all $n \geq 1, f_{n} \leq-\log \left(1-f_{n}\right)$ so that $f_{n} \leq \log s_{n}-\log s_{n-1}$ which implies that $\langle\mathcal{W}\rangle_{n+1} \leq \sigma^{2} \log s_{n}$. Hence, we deduce from the standard strong law of large numbers for martingales that $\mathcal{W}_{n+1}=o\left(\log s_{n}\right)$ a.s. Consequently, it immediately follows from (A.2) that $\mathcal{A}_{n}=o\left(\log s_{n}\right)$ a.s. However, we clearly have $f_{n}=a_{n}(1) v_{n}(1)$ with

$$
a_{n}(1)=\frac{\sqrt{s_{n}}+\sqrt{s_{n-1}}}{\sqrt{s_{n}}} .
$$

As $a_{n}(1) \rightarrow 2$ and $\mathcal{A}_{n}=o\left(\log s_{n}\right)$ a.s. it is not hard to see that a.s.

$$
\sum_{k=1}^{n} f_{k} V_{k}=o\left(\log s_{n}\right)+o\left(T_{n}\right) \quad \text { with } \quad T_{n}=\sum_{k=1}^{n} v_{k}(1)\left|V_{k}\right|
$$

Moreover, via the Cauchy-Schwarz inequality

$$
T_{n}^{2} \leq \sum_{k=1}^{n} f_{k} \sum_{k=1}^{n} f_{k} V_{k}^{2}
$$


because, for all $n \geq 1, a_{n}(1) \geq 1$ so $v_{n}(1) \leq f_{n}$. Furthermore, we can deduce from convergence (2.3) with $p=1$ that

$$
\sum_{k=1}^{n} f_{k} V_{k}^{2}=O\left(\log s_{n}\right) \quad \text { a.s. }
$$

Consequently, $T_{n}=O\left(\log s_{n}\right)$ a.s. which, by use of (A.4), clearly leads to (2.4) for $p=1$. Now, let $p \geq 2$ and assume that convergence (2.4) holds for any power $q$ with $1 \leq q \leq p-1$. We infer from formula (2.5) of [2] or formula (2.30) of [19] that $M_{n}^{2 p}=O\left(s_{n-1}^{p} \log s_{n-1}\right)$ a.s. so that $V_{n+1}=o\left(\log s_{n}\right)$ a.s. Next, we may assume without loss of generality that for all $n \geq 0, \sigma_{n}(2 p) \leq C$ a.s. for some constant $C \geq 1$. On the one hand, it follows from Chow's lemma (see e.g. Duflo [8] Theorem 1.3.18 p. 22) that

$$
\mathcal{W}_{n+1}=o\left(\sum_{k=1}^{n} f_{k}^{p}\right)+O\left(\sum_{k=1}^{n} f_{k}^{p-1 / 2}\right) \quad \text { a.s. }
$$

Hence, as $f_{n} \leq 1$ and $f_{n} \rightarrow 0$ a.s., we find that

$$
\mathcal{W}_{n+1}=o\left(\log s_{n}\right) \quad \text { a.s. }
$$

On the other hand, we also claim that

$$
\mathcal{B}_{n+1}=o\left(\log s_{n}\right) \quad \text { a.s. }
$$

In order to prove (A.6), it is only necessary to show that for any integer $1 \leq l \leq 2(p-1), B_{n+1}(l)=o\left(\log s_{n}\right)$ a.s. We can split $B_{n+1}(l)$ into two terms, $B_{n+1}(l)=C_{n+1}(l)+D_{n}(l)$ where

$$
C_{n+1}(l)=\sum_{k=1}^{n} \varphi_{k}(l) e_{k+1}(l) \quad \text { and } \quad D_{n}(l)=\sum_{k=1}^{n} \varphi_{k}(l) \sigma_{k}(l)
$$

with, for any $1 \leq l \leq 2(p-1), e_{k+1}(l)=\varepsilon_{k+1}^{l}-\sigma_{k}(l)$. First, for any $1 \leq l \leq p$, the sequence $\left(C_{n}(l)\right)$ is a locally square integrable martingale satisfying via the strong law of large numbers for martingales $\left|C_{n+1}(l)\right|^{2}=O\left(\tau_{n}(l) \log \tau_{n}(l)\right)$ a.s. where

$$
\tau_{n}(l)=\sum_{k=1}^{n}\left|\varphi_{k}(l)\right|^{2} .
$$

Moreover, one can easily deduce from formulas (2.5) and (2.6) of [2] that $\tau_{n}(l)=O\left(\left(\log s_{n}\right)^{d}\right)$ a.s. with $d=2(p-1) / p$. Consequently, as $d<2$, we immediately obtain that for any $1 \leq l \leq p$,

$$
C_{n+1}(l)=o\left(\log s_{n}\right) \quad \text { a.s. }
$$


Next, for $p \geq 3$ and for any $p+1 \leq l \leq 2(p-1)$, we find via Chow's lemma that either $\left(C_{n}(l)\right)$ converges a.s. or $C_{n+1}(l)=o\left(\nu_{n}(l)\right)$ a.s. where

$$
\nu_{n}(l)=\sum_{k=1}^{n}\left|\varphi_{k}(l)\right|^{\delta} \leq \sum_{k=1}^{n} f_{k}^{p}\left(\frac{M_{k}^{2}}{s_{k-1}}\right)^{\rho}
$$

with $\delta=2 p / l$ and $2 \rho=p(\delta-1)-\delta$. Since $p \geq 3$, we obviously have $1<\delta<2$ and $0<\rho<p$. In addition, it follows from the Hölder inequality that

$$
\nu_{n}(l) \leq\left(\sum_{k=1}^{n} f_{k}^{p}\right)^{1-\rho / p}\left(\sum_{k=1}^{n} f_{k}^{p}\left(\frac{M_{k}^{2}}{s_{k-1}}\right)^{p}\right)^{\rho / p} .
$$

Hence, as $f_{n} \rightarrow 0$ a.s., we infer from $(2.3)$ that $\nu_{n}(l)=o\left(\log s_{n}\right)$ so that (A.7) holds fo any $1 \leq l \leq 2(p-1)$. In order to prove (A.6), as $D_{n}(1)=0$, it remains to show that for any $2 \leq l \leq 2(p-1)$

$$
D_{n}(l)=o\left(\log s_{n}\right) \quad \text { a.s. }
$$

One can easily see from the Hölder inequality that for each $2 \leq l \leq 2(p-1)$, $\left|\sigma_{n}(l)\right| \leq C$ a.s. Consequently, we find that for any $2 \leq l \leq 2(p-1)$

$$
\left|D_{n}(l)\right| \leq C \sum_{k=1}^{n} f_{k}^{l / 2}\left(\frac{M_{k}}{\sqrt{s_{k-1}}}\right)^{2 p-1-l} \quad \text { a.s. }
$$

We shall study the asymptotic behavior of $D_{n}(l)$ in the three following cases for proving (A.8).

Case 1. Let $l=2$. It follows from the induction assumption that for any integer $1 \leq q \leq p-1$

$$
\sum_{k=1}^{n} f_{k}\left(\frac{M_{k}}{\sqrt{s_{k-1}}}\right)^{2 q-1}=o\left(\log s_{n}\right) \quad \text { a.s. }
$$

By use of (A.10) with $q=p-1$, we obtain that

$$
D_{n}(2)=\sigma^{2} \sum_{k=1}^{n} f_{k}\left(1-f_{k}\right)^{p-3 / 2}\left(\frac{M_{k}}{\sqrt{s_{k-1}}}\right)^{2 p-3}=o\left(\log s_{n}\right) \quad \text { a.s. }
$$

Case 2. Assume that $4 \leq l \leq 2(p-1)$ with $l$ even. If $2 \leq p \leq 5$, we proceed exactly as in b). Next, if $p \geq 6$, we have to consider two cases.

a) If $4 \leq l \leq p-2$ with $l$ even, we can find $1 \leq q \leq p-5$ such that $q=p-l-1$. Hence, it follows from (A.9) together with the Cauchy-Schwarz inequality and (2.3) that

$$
\left|D_{n}(l)\right|=O\left(\sum_{k=1}^{n} f_{k}^{l-1}\left(\frac{M_{k}}{\sqrt{s_{k-1}}}\right)^{2 q} \sum_{k=1}^{n} f_{k}\left(\frac{M_{k}}{\sqrt{s_{k-1}}}\right)^{2 p}\right)^{1 / 2}=o\left(\log s_{n}\right) \quad \text { a.s. }
$$


b) If $p-1 \leq l \leq 2(p-1)$ with $l$ even, we can choose $1 \leq q \leq p$ such that $q=2 p-l-1$. Then, we deduce once again from (A.9) together with the Cauchy-Schwarz inequality and (2.3) that

$$
\left|D_{n}(l)\right|=O\left(\sum_{k=1}^{n} f_{k}^{l-1} \sum_{k=1}^{n} f_{k}\left(\frac{M_{k}}{\sqrt{s_{k-1}}}\right)^{2 q}\right)^{1 / 2}=o\left(\log s_{n}\right) \quad \text { a.s. }
$$

Case 3. Assume that $3 \leq l \leq 2 p-3$ with $l$ odd. Then, we can find $1 \leq q \leq p-2$ such that $2 q=2 p-l-1$. Consequently, we immediately obtain from (A.9) and (2.3) that

$$
\left|D_{n}(l)\right|=O\left(\sum_{k=1}^{n} f_{k}^{l / 2}\left(\frac{M_{k}}{\sqrt{s_{k-1}}}\right)^{2 q}\right)=o\left(\log s_{n}\right) \quad \text { a.s. }
$$

Therefore, (A.8) clearly follows from the above three cases. Finally, we find from (A.2) together with (A.5) and (A.6) that $\mathcal{A}_{n}=O\left(\log s_{n}\right)$ a.s. Furthermore, we have the decomposition $f_{n}=a_{n}(p) v_{n}(p)$ where $a_{n}(p)$ is given by

$$
a_{n}(p)=\frac{1-b_{n}^{2}}{1-b_{n}^{2 p-1}} \quad \text { with } \quad b_{n}=\frac{\sqrt{s_{n-1}}}{\sqrt{s_{n}}}
$$

As $b_{n}$ tends to 1 a.s., we obtain by use of L'Hopital's rule that $a_{n}(p)$ converges to $2 /(2 p-1)$ a.s. Whence, as $\mathcal{A}_{n}=o\left(\log s_{n}\right)$ a.s., it ensures that a.s.

$$
\sum_{k=1}^{n} f_{k} V_{k}=o\left(\log s_{n}\right)+o\left(T_{n}\right) \quad \text { with } \quad T_{n}=\sum_{k=1}^{n} v_{k}(p)\left|V_{k}\right|
$$

In addition, we obtain from the Hölder inequality

$$
T_{n} \leq\left(\sum_{k=1}^{n} v_{k}(p)\right)^{1 / 2 p}\left(\sum_{k=1}^{n} v_{k}(p)\left(\frac{M_{k}}{\sqrt{s_{k-1}}}\right)^{2 p}\right)^{1-1 / 2 p}
$$

However, by the convexity of the function $x^{p-1 / 2}$, we have for all $n \geq 1$ and for any $p \geq 2,2(2 p-1)^{-1} \leq a_{n}(p) \leq 1$ which implies that $v_{n}(p) \leq p f_{n}$ and

$$
T_{n} \leq p\left(\sum_{k=1}^{n} f_{k}\right)^{1 / 2 p}\left(\sum_{k=1}^{n} f_{k}\left(\frac{M_{k}}{\sqrt{s_{k-1}}}\right)^{2 p}\right)^{1-1 / 2 p}
$$

Finally, it follows from $(2.3)$ that $T_{n}=O\left(\log s_{n}\right)$ a.s. which, by use of (A.11), leads to convergence (2.4) completing the proof of Theorem 3. 


\section{Appendix B.}

Appendix B deals with the proof of Theorem 5. As in Appendix A, we shall only study convergence for odd moments (3.3) as convergence for even moments (3.2) was already established in [2]. We shall prove convergence (3.3) by induction on the power $p \geq 1$ with a repeated use of decomposition (A.2). For $p=1$, we already saw that $V_{n+1}^{2}=O\left(\log s_{n}\right)$ a.s. In addition, as the explosion coefficient $f_{n}$ converges a.s. to $f, s_{n-1} / s_{n}$ tends a.s. to $1-f$ and $\log s_{n}$ is a.s. equivalent to $-n \log (1-f)$. Consequently, we obtain that $V_{n+1}^{2}=O(n)$ which leads to $V_{n+1}=o(n)$ a.s. Moreover, $\left(\mathcal{W}_{n}\right)$ is a locally square integrable martingale with increasing process $\left(\langle\mathcal{W}\rangle_{n}\right)$ such that

$$
\lim _{n \rightarrow \infty} \frac{1}{n} \mathcal{W}_{n+1}=\sigma^{2} f \quad \text { a.s. }
$$

Hence, according to the standard strong law of large numbers for martingales $\mathcal{W}_{n+1}=o(n)$ a.s. Therefore, it clearly follows from (A.2) that $\mathcal{A}_{n}=o(n)$ a.s. Furthermore, as $f_{n} \rightarrow f$ a.s., $v_{n}(1)$ converges a.s. to $1-\sqrt{1-f}$. Consequently, we obtain that a.s.

$$
\sum_{k=1}^{n} V_{k}=o(n)+o\left(T_{n}\right) \quad \text { with } \quad T_{n}=\sum_{k=1}^{n}\left|V_{k}\right| .
$$

Moreover, it follows from the Cauchy-Schwarz inequality together with convergence (3.2) for $p=1$ that $T_{n}=O(n)$ a.s. Thus, (B.1) immediately implies (3.3) for $p=1$. Now, let $p \geq 2$ and assume that convergence (3.3) holds for any power $q$ with $1 \leq q \leq p-1$. We already saw in Appendix A that $V_{n+1}=o\left(\log s_{n}\right)$ so that $V_{n+1}=o(n)$ a.s. In addition, it follows from Chow's lemma that

$$
\mathcal{W}_{n+1}=o\left(\sum_{k=1}^{n} f_{k}^{p}\right)+O\left(\sum_{k=1}^{n} f_{k}^{p-1 / 2}\left|\sigma_{k}(2 p-1)\right|\right) \quad \text { a.s. }
$$

Hence, as $f_{n} \rightarrow f$ and $\sigma_{n}(2 p-1)$ tends to zero a.s., we deduce that

$$
\mathcal{W}_{n+1}=o(n) \quad \text { a.s. }
$$

Next, via the same reasoning as in Appendix A, we find that for any $1 \leq l \leq$ $2(p-1), C_{n+1}(l)=o(n)$ a.s which leads to

$$
\mathcal{B}_{n+1}=\sum_{l=2}^{2 p-2} C_{2 p-1}^{l} D_{n}(l)+o(n) \quad \text { a.s. }
$$

It remains to study the asymptotic behavior of $D_{n}(l)$ in the three following cases. 
Case 1. Let $l=2$. It follows from the induction assumption that for any integer $1 \leq q \leq p-1$

$$
\sum_{k=1}^{n}\left(\frac{M_{k}}{\sqrt{s_{k-1}}}\right)^{2 q-1}=o(n) \quad \text { a.s. }
$$

Then, we infer from (B.4) with $q=p-1$ that

$$
D_{n}(2)=\sigma^{2} \sum_{k=1}^{n} f_{k}\left(1-f_{k}\right)^{p-3 / 2}\left(\frac{M_{k}}{\sqrt{s_{k-1}}}\right)^{2 p-3}=o(n) \quad \text { a.s. }
$$

Case 2. Assume that $4 \leq l \leq 2(p-1)$ with $l$ even. We split $D_{n}(l)$ into two terms,

$$
D_{n}(l)=\sigma(l) \sum_{k=1}^{n} \varphi_{k}(l)+\sum_{k=1}^{n} \varphi_{k}(l)\left(\sigma_{k}(l)-\sigma(l)\right) .
$$

Moreover, we can find $1 \leq q \leq p-2$ such that such that $2 q=2 p-l$. Hence, we deduce from (B.4) that

$$
\sum_{k=1}^{n} \varphi_{k}(l)=\sum_{k=1}^{n} f_{k}^{p-q}\left(1-f_{k}\right)^{q-1 / 2}\left(\frac{M_{k}}{\sqrt{s_{k-1}}}\right)^{2 q-1}=o(n) \quad \text { a.s. }
$$

Furthermore, it follows from the Hölder inequality that

$$
\sum_{k=1}^{n}\left|\varphi_{k}(l)\right| \leq \sum_{k=1}^{n}\left(\frac{\left|M_{k}\right|}{\sqrt{s_{k-1}}}\right)^{2 q-1} \leq n^{\rho / p}\left(\sum_{k=1}^{n}\left(\frac{M_{k}}{\sqrt{s_{k-1}}}\right)^{2 p}\right)^{1-\rho / p}
$$

with $\rho=p-q+1 / 2$ which, via (3.2), ensures that

$$
\sum_{k=1}^{n}\left|\varphi_{k}(l)\right|=O(n) \quad \text { a.s. }
$$

Consequently, we obtain from (3.1) and (B.5) that

$$
D_{n}(l)=o(n)+o\left(\sum_{k=1}^{n}\left|\varphi_{k}(l)\right|\right)=o(n) \quad \text { a.s. }
$$

Case 3. Assume that $3 \leq l \leq 2 p-3$ with $l$ odd. Then, we can find $1 \leq q \leq p-2$ such that $2 q=2 p-l-1$ and we directly obtain from (3.2) that

$$
\sum_{k=1}^{n}\left|\varphi_{k}(l)\right|=O\left(\sum_{k=1}^{n}\left(\frac{M_{k}}{\sqrt{s_{k-1}}}\right)^{2 q}\right)=O(n) \quad \text { a.s. }
$$

Whence, as $\sigma_{n}(l) \rightarrow 0$ a.s., we infer that

$$
D_{n}(l)=O(1)+o\left(\sum_{k=1}^{n}\left|\varphi_{k}(l)\right|\right)=o(n) \quad \text { a.s. }
$$


According to the above three cases, we find that for any $2 \leq l \leq 2(p-1)$, $D_{n}(l)=o(n)$ a.s. and we immediately deduce from (B.3) that

$$
\mathcal{B}_{n+1}=o(n) \quad \text { a.s. }
$$

Consequently, it follows from the conjunction of (A.2), (B.2) and (B.6) that $\mathcal{A}_{n}=o(n)$ a.s. Finally, as $f_{n} \rightarrow f$ a.s., $v_{n}(p)$ converges a.s. to $1-(1-f)^{p-1 / 2}$ which ensures that

$$
\sum_{k=1}^{n} V_{k}=o(n) \quad \text { a.s. }
$$

completing the proof of Theorem 5 .

\section{References}

[1] Berkes I., Csáki E., 2001. A universal result in almost sure central limit theory. Stochastic Process. Appl. 94, 105-134.

[2] B. Bercu, 2004. On the convergence of moments in the almost sure central limit theorem for martingales with statistical applications. Stochastic Process. Appl. $111,157-173$.

[3] Billingsley P., Probability and measure, third edition John Wiley \& Sons, New York, 1995.

[4] Brosamler G. A., 1988. An almost sure everywhere central limit theorem. Math. Proc. Cambridge Philos. Soc. 104, 561-574.

[5] Chaabane F., 1996. Version forte du théorème de la limite centrale fonctionnel pour les martingales. C. R. Acad. Sci. Paris 323, 195-198.

[6] Chaabane F., Maaouia F., 2000. Théorèmes limites avec poids pour les martingales vectorielles. Esaim Prob. Stat. 4, 137-189.

[7] Chaabane F., 2001. Invariance principle with logarithm averaging for martingales. Studia Math. Sci. Hungar. 37, 21-52.

[8] Duflo M., 1997. Random Iterative Models, Springer Verlag, Berlin.

[9] Guttorp P., Statistical Inference for Branching Processes John Wiley, New York, 1991.

[10] Ibragimov I. A., Lifshits M. A., 1998. On the convergence of generalized moments in almost sure central limit theorem. Statist. Probab. Lett. 40, 343351.

[11] Ibragimov I. A., Lifshits M. A., 1999. On almost sure limit theorems. Theory Probab. Appl. 44, 254-272.

[12] Lacey, M. T., Phillip W., 1990. A note on the almost sure central limit theorem. Statist. Probab. Lett. 9, 201-205. 
[13] Lai T. L., Wei C. Z., 1982. Least squares estimates in stochastic regression models with applications to identification and control of dynamic systems. Ann. Statist. 10, 154-166.

[14] Lifshits M., 2002. Almost sure limit theorem for martingales. Limit Theorems in Probability and Statistics II, (I. Berkes, E. Csáki, M. Csörgő, eds.) J. Bolyai Mathematical Society, Budapest, 367-390.

[15] Lifshits M., 2001. Lecture notes on almost sure limit theorems, Publications IRMA 54, Lille, 1-25.

[16] Maaouia F., Touati A.,, 2005. Identification of multitype branching processes. To appear in Ann. Statist.

[17] Schatte, P., 1988. On strong versions of the almost sure central limit theorem. Math Nachr. 137, 249-256.

[18] Schatte, P., 1991. On the central limit theorem with almost sure convergence. Probab. Math. Statist. 11, 237-246.

[19] Wei C. Z., 1987. Adaptive prediction by least squares predictors in stochastic regression models with applications to time series. Ann. Statist. 15, 1667-1682. 